\title{
Pilot Study of the Influence of Eyeliner Cosmetics on the Molecular Structure of Human Meibum
}

\author{
Morgan Hunter $^{\mathrm{a}}$ Rahul Bhola ${ }^{\mathrm{b}}$ Marta C. Yappert ${ }^{\mathrm{a}} \quad$ Douglas Borchman $^{\mathrm{b}}$ \\ Dylan Gerlach ${ }^{\mathrm{b}}$ \\ Departments of a Chemistry and ${ }^{b}$ Ophthalmology and Visual Sciences, University of Louisville, Louisville, Ky., USA
}

\author{
Key Words \\ Dry eye $\cdot$ Infrared spectroscopy $\cdot$ Lipids · Makeup $\cdot$ Meibum
}

\begin{abstract}
Background/Aims: It has been suggested that eye makeup could interact with human meibum causing a decrease in the stability of the tear film. The aim of this pilot study was to measure makeup-human meibum interactions in vitro. Methods: Human meibum-makeup interactions were quantified by measuring order-to-disorder lipid phase transitions using infrared spectroscopy. Results: Makeup products exhibited lipid phase transition temperatures that were much higher than those for meibum. One product increased the lipid phase transition temperature by $4.2^{\circ} \mathrm{C}$ when combined with human meibum causing a large increase (from 30 to $49 \%$ ) in the order of the meibum-lipid hydrocarbon chains and significantly decreased the minimum frequency, enthalpy and entropy of the phase transition of human meibum. Another eyeliner caused no significant $(p<0.05)$ change in the phase transition parameters of human meibum. Conclusion: Infrared spectroscopy may be used to measure interactions between human meibum and makeup. One makeup product increased the lipid order (viscosity) which could have adverse effects on tear film stability. Modern cosmetics
\end{abstract}

are highly regulated and relatively safe to use; however, it could be beneficial to design makeup products that do not interact with meibum, especially since women have a higher prevalence of dry eye symptoms.

(C) 2015 S. Karger AG, Base

\section{Introduction}

Women and men have painted their eyes since prehistoric Paleolithic and neolithic times [1]. Today, mascara is applied to the eyelashes to color them. Mineral, linseed, castor, eucalyptus, lanolin, turpentine, sesame oils and waxes such as paraffin, carnauba wax or beeswax are commonly mixed with iron oxides for color [2]. Reports of poisoning in Arabic cultures from the use of lead-containing black eye paste have been reported [3]. Modern cosmetics are highly regulated and relatively safe to use [2]. A specular reflection device indicated that moisturizers used to remove cosmetics appeared as oily floaters or oily plaques on the surface of the eye, and it has been suggested that eyeliners, eye shadow and mascara could block meibomian glands, contaminate the meibum and increase the debris levels on the superficial lid layer [4]. Indeed, lipophilic substances applied to the lower eyelid

\section{KARGER 125}

(C) 2015 S. Karger AG, Basel

0030-3747/15/0533-0131\$39.50/0

E-Mail karger@karger.com

www.karger.com/ore
Rahul Bhola, MD

Kentucky Lions Eye Center

301 E Muhammad Ali Blvd

Louisville, KY 40202 (USA)

E-Mail r0bhol01@exchange.louisville.edu 
skin are able to reach the inferior tear meniscus supracutaneously and mix with the tear film lipid layer $[5,6]$. Maintenance of meibum structure may be critical to normal tear film function [7].

Dry eye is a multifactorial disease that is associated with elevated tear osmolarity and ocular irritation. It is one of the most common causes for patients to consult with an eye care professional [8]. It is more prevalent in women $[9,10]$ and, as suggested but not tested [4], could be exacerbated by eye makeup.

\section{Materials and Methods}

The work described has been carried out in accordance with the Code of Ethics of the World Medical Association (Declaration of Helsinki) for experiments involving humans. In this study, two popular cosmetics were mixed with meibum to test if they altered the molecular structure of human meibum. Meibum was collected and pooled [11] from 7 donors present in Professor Borchman's laboratory at the time of the study: MA21, FC21, FB21, MC59, MC22, FA42 and FA19, where $M$ is male, $F$ is female, $A$ is Asian, $C$ is Caucasian, $\mathrm{B}$ is black and the number is the age of the donor in years. The donors did not complain of dry eye symptoms or have any ocular diseases. Written informed consent was obtained from all donors. Protocols and procedures were reviewed by the institutional review boards of the University of Louisville. Stock $(1 \mathrm{mg} / \mathrm{ml}) \mathrm{CHCl}_{3}$ solutions of meibum alone, and meibum mixed with 25 weight percent makeup were dried onto a $\mathrm{AgCl}$ window and lyophilized for infrared spectroscopic analysis. Two popular brands of eyeliner makeup were studied: L'Oréal (Clichy, Hauts-de-Seine, France) Infallible pencil eyeliner and water-based Revlon (New York, N.Y., USA) ColorStay liquid eyeliner. The L'Oréal product contains over 20 ingredients including cyclopentasiloxane, hydrogenated olive oil esters, candelilla wax, microcristalline wax, sodium hyaluronate as well as iron oxides, titanium dioxide and ferric ferrocyanide. The Revlon product also contains over 20 ingredients such as water, octylacrylamide copolymer, Aloe barbadensis leaf juice, imidazolidinyl urea, methylparaben, ethylparaben, iron oxides and Black 2.

Infrared spectroscopy was used to measure and quantify the phase transitions of lipids $[11,12]$. The lipid hydrocarbon chain conformational order may be evaluated in terms of the amount of $\mathrm{CH}_{2}$ trans- and gauche rotamers. Conformation is how molecules are arranged in space. The methylene moieties may arrange as gauche rotamers, prevalent in disordered lipids like olive oil, which is fluid at room temperature. The methylene moieties may also arrange as trans-rotamers, more abundant in ordered lipids like butter, which is solid at room temperature.

Meibum and meibum-eyeliner samples were mixed in deuterium oxide and deuterated chloroform solvents for nuclear magnetic resonance (NMR) analysis in a Varian VNMR 700- $\mathrm{MHz}$ NMR spectrometer (Varian, Lexington, Mass., USA) equipped with a $5-\mathrm{mm}{ }^{1} \mathrm{H}\left[{ }^{13} \mathrm{C} /{ }^{15} \mathrm{~N}\right]{ }^{13} \mathrm{C}$-enhanced cold probe (Palo Alto, Calif., USA).

Data are presented as the average \pm the standard error of the mean unless indicated otherwise. Differences were compared using Student's $t$ test, and a $\mathrm{p}<0.05$ was considered statistically significant.

\section{Results}

The amount of makeup applied to one eyelid was estimated gravimetrically by quantifying the amount of makeup applied onto a $30 \times 1 \mathrm{~mm}$ area of paper. The average and standard deviation of the amount of L'Oréal and Revlon products applied was $0.0010 \pm 0.0002$ and $0.00305 \pm 0.0005 \mathrm{~g}$, respectively.

The phase transitions for meibum were reproducible, and the phase transition parameters were similar to those obtained previously for normal human meibum (fig. 1a) [11]. The L'Oréal product significantly increased $(\mathrm{p}<$ $0.05)$ the phase transition temperature of meibum lipid by $4.2^{\circ} \mathrm{C}$ (fig. $1 \mathrm{~b}$, table 1 ). Because the lipid phase transition temperature of human meibum is near the physiological temperature of the surface of the eye, the small increase in lipid phase transition temperature caused a large increase, from 30 to $49 \%$, in the order or stiffness of the meibum lipid hydrocarbon chains. The L'Oréal product also significantly $(\mathrm{p}<0.05)$ decreased the minimum frequency, enthalpy and entropy of the phase transition of human meibum (table 1). The Revlon product caused no significant $(p>0.05)$ change in the phase transition parameters of human meibum (fig. 1c, table 1).

Because of the high optical opacity of the Revlon product, very little infrared light was transmitted making it technically difficult to measure an exact phase transition of the Revlon product alone. Regardless of the technical difficulty, we observed that both the L'Oréal and Revlon products alone exhibited phase transitions that were at much higher phase transition temperatures than for meibum (fig. 1d, table 1). Broadening of the $-\mathrm{CH}_{2}-$ methylene resonances near $1.24 \mathrm{ppm}$ [13] confirmed the interaction of the L'Oréal eyeliner with meibum (fig. 2). The Revlon eyeliner showed little interaction with meibum supporting the infrared spectroscopy phase transition data. We also studied the interaction of the wax palmityl oleate with the two makeup products. Palmityl oleate was chosen because it has a phase transition at a temperature which could be conveniently measured, $13.1 \pm 0.1^{\circ} \mathrm{C}$. The phase transition increased significantly ( $\mathrm{p}<0.0001)$ to $14.08 \pm 0.07$ and $15.32 \pm 0.07^{\circ} \mathrm{C}$ when the palmityl oleate was mixed at $25 \%$ by weight with the Revlon and L'Oréal products, respectively.

\section{Discussion}

We quantified meibum-makeup interactions in a $25 \%$ by weight mixture. Concentrations of makeup much higher than this could overwhelm the infrared bands of 


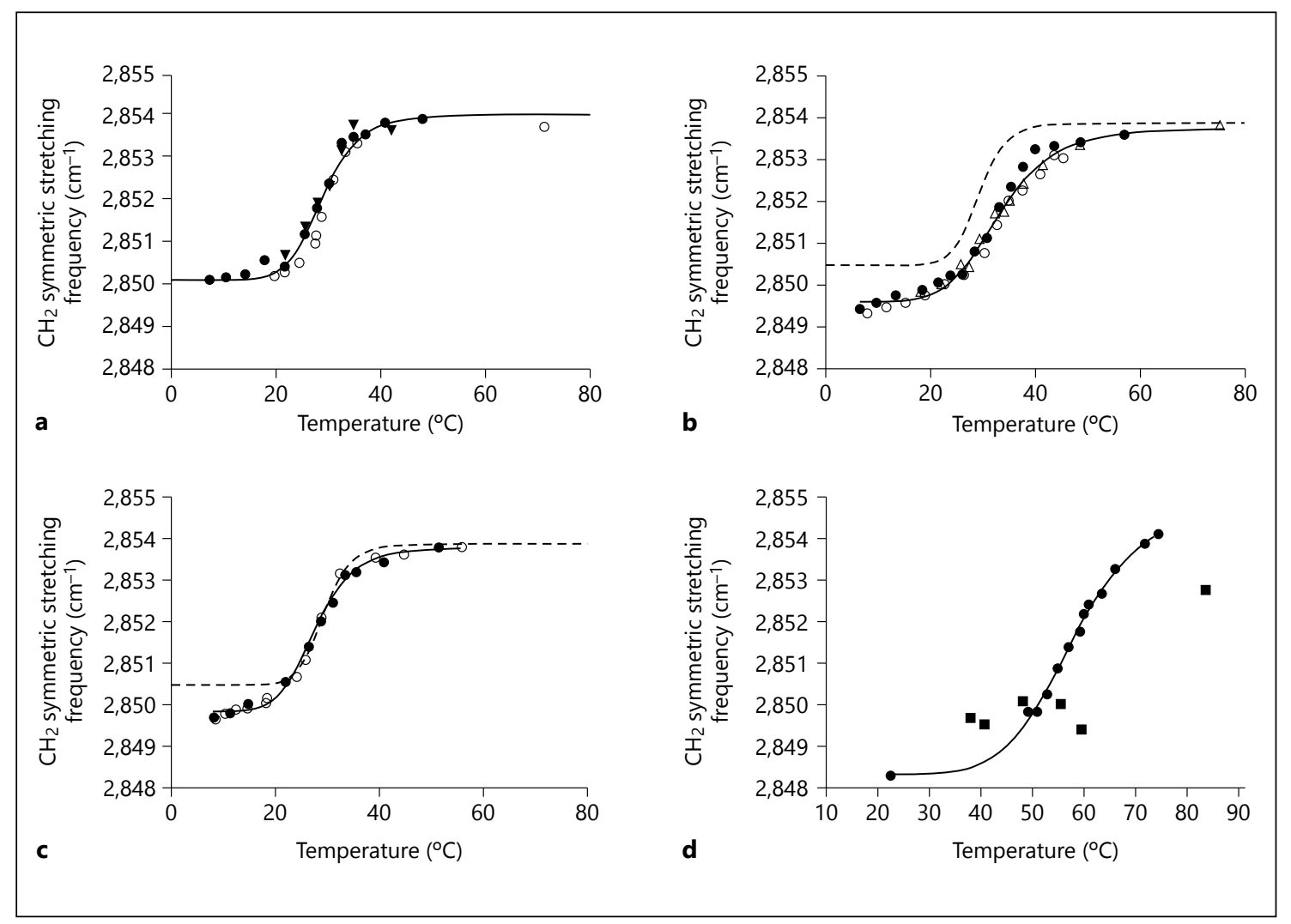

Fig. 1. Lipid phase transition curves measured using infrared spectroscopy. The lower the $\mathrm{CH}_{2}$ symmetric stretching frequency, the more ordered the wax hydrocarbon chains. Lines are the fit of the data to equation 1 in Borchman et al. [12]. Symbols are for experiments conducted on different days. a Human meibum. b Human meibum plus L'Oréal Infallible pencil eyeliner (25\% by weight).
- - - = Curve fit of meibum-only data from a. c Human meibum plus water-based Revlon ColorStay liquid eyeliner (25\% by weight). $--_{-}=$Curve fit of meibum-only data from a. d Comparison of L'Oréal Infallible pencil eyeliner $(\bullet)$ and water-based Revlon ColorStay liquid eyeliner ( $\mathbf{\square})$.

Table 1. Lipid phase transition parameters

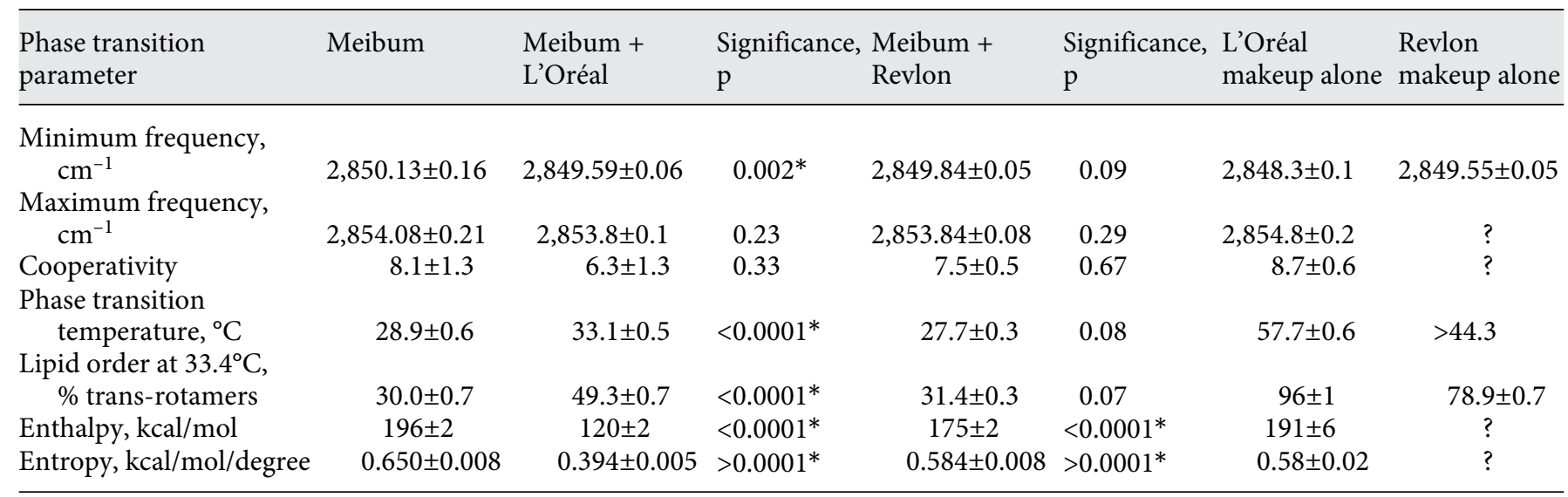

Eyeliners: L’Oréal Infallible pencil eyeliner and water-based Revlon ColorStay liquid eyeliner. ${ }^{*} \mathrm{p}<0.05$ : statistically significant, when makeup was mixed with meibum ( $25 \%$ by weight). Data are the average \pm the standard deviation of the mean. $p$ values were determined using Student's t test. 


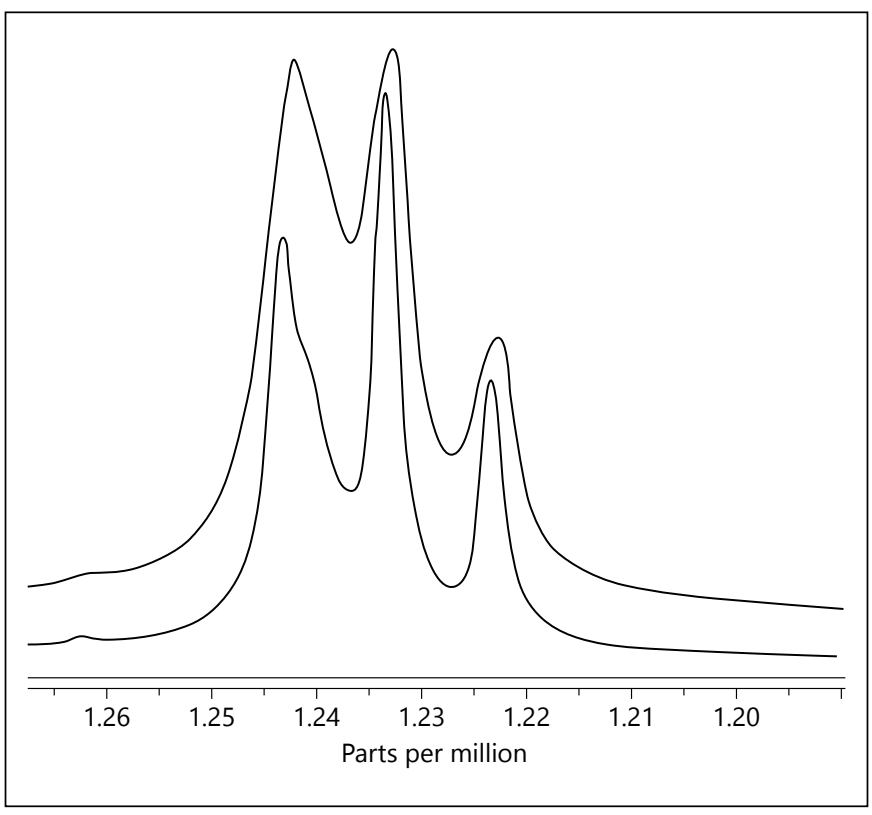

Fig. 2. ${ }^{1} \mathrm{H}-\mathrm{NMR}$ spectra of human meibum (bottom) and human meibum with L'Oréal Infallible pencil eyeliner (top; $25 \%$ by weight).

meibum, interfering with the quantification of meibum lipid conformation. Concentrations of makeup much lower than the 3:1 (meibum:makeup) ratio we used (25\%) would not be physiologically relevant since we calculate that the makeup:meibum ratio on the eyelid surface is much higher, 1:10 (91\%) to 1:70 (99\%), based on the amount of casual meibum on the surface of the eyelid [14].

Infrared spectroscopy was used in the current study to measure lipid-makeup interactions with the hydrocarbon chains. The conformation of hydrocarbon chains of human meibum lipids is in between that of fluid-like lipids such as olive oil and/or solid-like lipids such as butter [15]. Fluid lipids are generally not completely liquid, but interact with one another and are said to be in a gel phase. Solid lipids are not completely solid and contain some mobility so they are said to be in a liquid crystalline phase. We found that one makeup product caused the hydrocarbon chains of meibum to become more 'solid' and in a gel phase where the hydrocarbons pack tightly together and van der Waals' interactions between hydrocarbon chains are maximal. When the lipids were more fluid and in a liquid crystalline phase, gauche rotamers caused the hydrocarbon chains to bend. The surface area of fluid lipids was larger than that of ordered lipids, and van der Waals' interaction between chains was minimal. In simpler terms, one makeup product caused the meibum to become more viscous.

Meibum lipid hydrocarbon chains interact with each other via hydrophobic van der Waal's interactions [11, 12]. Cations, which interact with lipids ionically, do not cause a change in human meibum packing [16]. Therefore, we speculate that the reason why the Revlon-based product did not cause a change in meibum conformation is that the Revlon product is water based and insoluble in solvents such as chloroform. Therefore, the hydrophilic ingredients did not interact with the hydrophobic chains of the meibum. The L'Oréal product contains hydrophobic moieties such as cyclopentasiloxane, hydrogenated olive oil esters, candelilla wax and microcristalline wax. The acyl chain esters are all saturated and very ordered. We found that saturation is the major factor that contributes to hydrocarbon chain order $[11,12]$ and that when a saturated wax was mixed with an unsaturated one, the saturated wax disproportionately increased the phase transition of the mixture by approximately $30^{\circ} \mathrm{C}$ compared with the saturated wax alone [11]. The mixing of the saturated waxes with meibum could easily account for the small $3^{\circ} \mathrm{C}$ change in the phase transition temperature observed when the L'Oréal eyeliner was mixed with meibum or synthetic wax. It is doubtful that the $\mathrm{CH}_{3}$ moieties of cyclopentasiloxane interact with the $\mathrm{CH}_{2}$ hydrocarbon groups of meibum.

We used the synthetic wax palmityl oleate to study the interaction with makeup. Waxes are the predominant lipid species of meibum [7]. At present it is difficult to make a mixture of synthetic lipids as a good model for meibum because meibum contains over '30,000 molecular species' [17] of lipid and the composition is complicated by branched chains, unsaturation and hydroxylation and products such as (O-acyl)- $\dot{\omega}$-hydroxy fatty acids that are not available commercially [7]. The wax palmityl oleate may be used to screen products as we did. We found that the L'Oréal product increased the phase transition temperature of palmityl oleate more effectively than the Revlon product similarly to the effect it had on meibum. Ultimately, because of the complexity of the human meibum lipidome, it is important to use human meibum to test makeup.

As a pilot study, one must be careful to draw conclusions about the potential functional results of altering the phase transition parameters of human meibum. The phase transition temperature of meibum from donors with dry eye due to meibomian gland dysfunction is higher than that of meibum from normal donors [11], similarly to the lipid phase transition-rising effect of the 
L'Oréal product. Maintenance of the lipid phase transition temperature close to the physiological temperature may be critical to the maintenance of a low evaporation rate [18]. Lipid order (viscosity or stiffness) has been related to the release of meibum from the meibomian glands [19]. Makeup carried with meibum onto the surface of the eye could also affect the surface properties of the lipid layer of the tear film. There is about 33 times more meibum lipid on the eyelid surface compared with the layer of meibum lipid on the surface of tears, so contamination of the tear film could be more critical than contamination of the eyelid surface [14]. Eye makeup is designed to stick to the skin and is naturally hydrophobic. Modern cosmetics are highly regulated and relatively safe to use; however, it could be beneficial to design makeup products that do not interact with meibum, especially since women have a higher prevalence of dry eye symp- toms $[9,10]$. Eye makeup could even be beneficial if ingredients that enhance tear film stability were included in the formulations.

\section{Acknowledgments}

This work was supported by the Kentucky Lions Eye Foundation and an unrestricted grant from Research to Prevent Blindness Inc. Morgan Hunter's fellowship with the Department of Chemistry at the University of Louisville was funded by the National Science Foundation Research Experience for Undergraduates. Dylan Gerlach's fellowship was funded by the Nation Institute of Health, Kentucky Biomedical Research Infrastructure Network grant.

\section{Disclosure Statement}

None of the authors had a conflict of interest.

\section{References}

$>1$ Murube J: Ocular cosmetics in ancient times. Ocul Surf 2013;11:2-7.

$\checkmark 2$ Murube J: Ocular cosmetics in modern times. Ocul Surf 2013;11:60-64.

$>3$ De Caluwé JP: Saturnine intoxication from the long-term use of kohl: an underestimated cause in French-speaking countries (in French). J Fr Ophtalmol 2009;32:459-463.

4 Guillon JP: Abnormal lipid layers. Observation, differential diagnosis, and classification. Adv Exp Med Biol 1998;438:309-313.

$>5$ Tsubota K, Monden Y, Yagi Y, Goto E, Shimmura S: New treatment of dry eye: the effect of calcium ointment through eyelid skin delivery. Br J Ophthalmol 1999;83:767770.

6 Goto E, Dogru M, Fukagawa K, Uchino M, Matsumoto Y, Saiki M, Tsubota K: Successful tear lipid layer treatment for refractory dry eye in office workers by low-dose lipid application on the full-length eyelid margin. Am J Ophthalmol 2006;142:264-270.

-7 Green-Church KB, Butovich I, Willcox M, Borchman D, Paulsen F, Barabino S, Glasgow BJ: The international workshop on meibomian gland dysfunction: report of the subcommittee on tear film lipids and lipid-protein interactions in health and disease. Invest Ophthalmol Vis Sci 2011;52:1979-1993.
8 Schaumberg DA, Sullivan DA, Dana MR: Epidemiology of dry eye syndrome. Adv Exp Med Biol 2002;506:989-998.

$>9$ Pflugfelder SC: Prevalence, burden, and pharmacoeconomics of dry eye disease. Am J Manag Care 2008;14(suppl):S102-S106.

$>10$ Schaumberg DA, Nichols JJ, Papas EB, Tong L, Uchino M, Nichols KK: The International Workshop on Meibomian Gland Dysfunction: report of the subcommittee on the epidemiology of, and associated risk factors for, MGD. Invest Ophthalmol Vis Sci 2011;52: 1994-2005.

-11 Borchman D, Foulks GN, Yappert MC, Bell J, Wells E, Neravetla S, Greenstone V: Human meibum lipid conformation and thermodynamic changes with meibomian-gland dysfunction. Invest Ophthalmol Vis Sci 2011;52: 3805-3817.

12 Borchman D, Foulks GN, Yappert MC, Tang D, Ho DV: Temperature-induced conformational changes in human tear lipids hydrocarbon chains. Biopolymers Biospectrosc 2007; 87:124-133.
13 Borchman D, Yappert MC, Milliner S, Duran D, Cox GW, Smith RJ, Bhola R: ${ }^{13} \mathrm{C}$ and ${ }^{1} \mathrm{H}$ $\mathrm{NMR}$ ester region resonance assignments and the composition of human infant and child meibum. Exp Eye Res 2013;112:151-159.

14 Chew CKS, Hykin PG, Janswijer C, Dikstein S, Tiffany JM, Bron AJ: The casual level of meibomian lipids in humans. Curr Eye Res 1993;12:255-259.

15 Borchman D: From the bench to the bedside. Infrared spectroscopy and the diagnosis and treatment of dry eye and cataracts. Spectroscopy 2014;29:38-52.

16 Wei XE, Markoulli M, Millar TJ, Willcox MD, Zhao Z: Divalent cations in tears, and their influence on tear film stability in humans and rabbits. Invest Ophthalmol Vis Sci 2012;53: 3280-3285.

17 Nicolaides N, Santos EC: The di- and triesters of the lipids of steer and human meibomian glands. Lipids 1985;20:454-467.

18 Rantamäki AH, Wiedmer SK, Holopainen JM: Melting points - the key to the anti-evaporative effect of the tear film wax esters. Invest Ophthalmol Vis Sci 2013;54:5211-5217.

19 Ashraf Z, Pasha U, Greenstone V, Akbar J, Borchman D: Quantification of human sebum on skin and human meibum on the eyelid margin using Sebutape ${ }^{\circledR}$, spectroscopy and chemical analysis. Curr Eye Res 2011;36:553-562. 